\title{
"Energy : A challenge for 2lst century physics" Une école européenne passionnante aux Houches
}

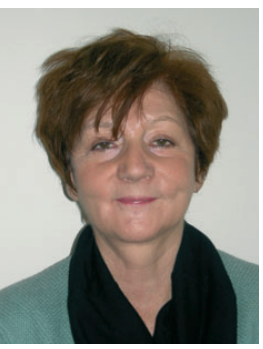

Une rencontre originale s'est déroulée aux Houches du 2 au 5 juin 2008. Organisée conjointement par Jean-Louis Bobin pour la SFP et par le président de l'EPS Fritz Wagner, elle a réuni des participants de plusieurs pays européens sur le thème multidisciplinaire de l'avenir énergétique de la planète. Elle a bénéficié d'un financement d'EDF, concrétisé par la venue de son directeur de la recherche Yves Bamberger. Sa brillante conférence : "More electricity for less $\mathrm{CO}_{2}$ : hints for research" a bien résumé l'ensemble des thèmes abordés par les intervenants, qui ont cherché à cerner les domaines de physique où la recherche est susceptible d'apporter des contributions significatives. La présence des Allemands à côté des Français a introduit un élément stimulant de compétition entre les scénarios proposés (" avec ou sans énergie nucléaire? ") dans un contexte marqué par la demande croissante d'énergie, la limitation des ressources naturelles, et les contraintes liées à l'environnement et au réchauffement de la planète.

Une revue des réserves en pétrole, gaz, charbon et uranium a d'abord indiqué que l'on pourrait disposer de ressources suffisantes pendant encore 100 ans si l'on faisait abstraction de toute considération de coût, d'émission de $\mathrm{CO}_{2}$, de facteurs géopolitiques et d'acceptation par les populations. II apparaît que la consommation en électricité d'un pays en émergence est strictement proportionnelle à l'accroissement de son PIB. Le charbon va de ce fait jouer un rôle croissant. Les centrales à charbon peuvent améliorer leurs rendements et diminuer leurs émissions de $\mathrm{CO}_{2}$ en utilisant simultanément du gaz ou de la biomasse. Le stockage et l'enfouissement $\mathrm{du} \mathrm{CO}_{2}$ restent très débattus (coût, limites de l'espace géologique, sécurité et acceptabilité par le public). L'énergie du vent, source intermittente, requiert encore beaucoup de recherches sur l'aérodynamique et surtout l'amélioration des prédictions météorologiques par de nouveaux satellites. La biomasse pourrait éviter certaines critiques en se concentrant sur les déchets des plantes cultivées pour l'alimentation. Le photovoltaïque est en plein progrès avec les multijonctions, le confinement optique, le silicium amorphe dopé ou encore les matériaux organiques. Les recherches sur le cycle de l'hydrogène sont interdisciplinaires, il faut résoudre les problèmes de dynamique de l'eau à travers les membranes et de diffusion du gaz vers les électrodes; elles devraient être coordonnées au niveau européen. Pour l'énergie nucléaire, les efforts actuels portent sur l'amélioration de la sécurité ; la quatrième génération a de nombreuses filières possibles, dont seulement deux sont à l'étude. La distribution de l'électricité est un problème central pour lequel l'optimisation des réseaux fait l'objet de recherches poussées : une "supergrid" en Europe est un enjeu crucial ; une extension jusqu'en Afrique est même envisagée. On rêve à la découverte de la supraconductivité à température ambiante. Quant aux économies d'énergie, elles requièrent aussi de la recherche sur les matériaux pour isoler les habitations; certains modèles suggèrent des méthodes pour réguler les flux de véhicules sur les routes, d'autres pour réduire considérablement le coût du trafic aérien.

Les recommandations de l'Union Européenne sont de limiter à $2^{\circ} \mathrm{C}$ l'augmentation de la température d'ici 2020, tout en diminuant de $20 \%$ les émissions de $\mathrm{CO}_{2}$, au prix d'un investissement annuel de $1 \%$ du PIB par pays. Toutefois, aucun des scénarios envisagés n'est totalement convaincant ; la solution passe évidemment par des décisions politiques à l'échelle de la planète. Les conclusions du débat à l'école des Houches pourront servir de base pour des recommandations en matière de recherche et d'enseignement pour l'énergie. L'impression générale que j'ai retirée personnellement de cette rencontre est qu'on n'a pas encore pris en France la mesure du travail à accomplir.

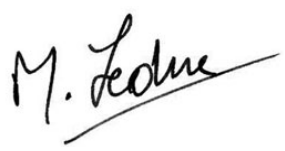

Michèle Leduc

Les présentations des conférenciers sont disponibles sur: www.sfpnet.fr/front_office/actu_en_detail.php?id_actu=297 